\title{
A REFINEMENT OF YOUNG'S INEQUALITY
}

\author{
P. KÓRUS \\ Department of Mathematics, Juhász Gyula Faculty of Education, University of Szeged, \\ Hattyas utca 10, 6725 Szeged, Hungary \\ e-mail: korpet@jgypk.u-szeged.hu
}

(Received April 3, 2017; accepted April 7, 2017)

\begin{abstract}
We present an improved version of Young's inequality as well as an operator inequality version of it. Our result is compared to the latest refinements.
\end{abstract}

\section{Introduction}

Throughout this paper let $a, b$ be arbitrary positive numbers and $0 \leq p$ $\leq 1$. Young's inequality or $p$-weighted arithmetic-geometric mean inequality says

$$
a^{p} b^{1-p} \leq p a+(1-p) b .
$$

During the past years, several refinements were given for Young's inequality, see for example [1].

In [2], the following inequality was proved by S. Furuichi:

$$
S\left(\left(\frac{b}{a}\right)^{r}\right) a^{p} b^{1-p} \leq p a+(1-p) b,
$$

where $r=\min \{p, 1-p\}$ and $S(x)$ is Specht's ratio (see [3]).

In [5], it was seen that

$$
K^{r}\left(\frac{b}{a}\right) a^{p} b^{1-p} \leq p a+(1-p) b,
$$

where $r=\min \{p, 1-p\}$ and $K(x)=\frac{(1+x)^{2}}{4 x}$ is Kantorovich's constant. It was also proved that $S\left(x^{s}\right) \leq K^{s}(x)$ for $x>0,0 \leq s \leq 1 / 2$, which means that (1.1) is a consequence of $(1.2)$.

Key words and phrases: Young's inequality, arithmetic-geometric mean inequality, operator inequality.

Mathematics Subject Classification: 26D07, 26D15, 47A63. 
In the special case when $p=1 / 2$, Young's inequality is the well-known arithmetic-geometric mean inequality

$$
\sqrt{a b} \leq \frac{a+b}{2} .
$$

In [4], an improved version of the arithmetic-geometric mean inequality was proved by L. Zou and Y. Jiang:

$$
\left(1+\frac{(\log a-\log b)^{2}}{8}\right) \sqrt{a b} \leq \frac{a+b}{2},
$$

moreover, the relationship between $S(\sqrt{b / a})$ and $1+\frac{(\log a-\log b)^{2}}{8}$ was discussed. While inequality (1.3) was proved in [4], we now give an alternative proof, which inspired the proof of our main result presented later.

Proof of (1.3). Without loss of generality, we can assume that $a \geq b$. Then (1.3) is equivalent to

$$
\left(1+\frac{(\log a-\log b)^{2}}{8}\right) \sqrt{\frac{a}{b}} \leq \frac{\frac{a}{b}+1}{2} .
$$

Using a substitution $x=\frac{a}{b} \geq 1$, we need that

$$
\left(1+\frac{\log ^{2} x}{8}\right) \sqrt{x} \leq \frac{x+1}{2},
$$

which is equivalent to

$$
\frac{\log ^{2} x}{4} \leq \frac{x-2 \sqrt{x}+1}{\sqrt{x}} .
$$

Now substituting $y=\sqrt{x} \geq 1$, the needed relation is

$$
\log ^{2} y \leq \frac{(y-1)^{2}}{y}
$$

which is equivalent to

$$
\log y \leq \frac{(y-1)}{\sqrt{y}} .
$$

This relation was proved in [2, Lemma 2].

However, the relationship between $K^{1 / 2}\left(\frac{b}{a}\right)$ and $1+\frac{(\log a-\log b)^{2}}{8}$ was not discussed in [4]. Now we prove that

$$
K^{1 / 2}(x) \geq 1+\frac{\log ^{2} x}{8} \quad \text { for } x>0,
$$


which means that we can obtain (1.3) from (1.2).

Proof of (1.4). Let

$$
f(x):=\frac{1+x}{2 \sqrt{x}}-1-\frac{\log ^{2} x}{8} .
$$

We need to see that $f(x) \geq 0$ on $(0, \infty)$. Since $f(1)=0$ and $f(x)$ is differentiable on $(0, \infty)$, it is enough to see that $f^{\prime}(x) \leq 0$ on $(0,1)$ and $f^{\prime}(x) \geq 0$ on $(1, \infty)$. As

$$
f^{\prime}(x)=\frac{x-1}{4 x \sqrt{x}}-\frac{\log x}{4 x},
$$

it is enough to see that the derivative of

$$
g(x):=4 x f^{\prime}(x)=\sqrt{x}-\frac{1}{\sqrt{x}}-\log x
$$

is non-negative, that is

$$
g^{\prime}(x)=\frac{x-2 \sqrt{x}+1}{2 x \sqrt{x}} \geq 0 \quad \text { for } x>0,
$$

which inequality holds.

\section{Main results}

We prove an inequality similar to (1.1) and (1.2), meanwhile we generalize (1.3). That is,

$$
\left(1+Q(p)(\log a-\log b)^{2}\right) a^{p} b^{1-p} \leq p a+(1-p) b,
$$

where $Q(p)=\frac{p^{2}}{2}\left(\frac{1-p}{p}\right)^{2 p}$ for $0<p<1$ and $Q(0)=Q(1)=0$.

Proof of (2.1). We can suppose that $0<p<1$. A division by $b$ and a substitution $x=\frac{a}{b}$ imply that we need to see

$$
\left(1+Q(p) \log ^{2} x\right) x^{p} \leq p x+1-p .
$$

Hence if we set

$$
f(x):=p x^{1-p}+(1-p) x^{-p}-1-Q(p) \log ^{2} x,
$$


then it is enough to prove that $f(x) \geq 0$ on $(0, \infty)$. Since $f(1)=0$ and $f(x)$ is differentiable on $(0, \infty)$, it is enough to see that $f^{\prime}(x) \leq 0$ on $(0,1)$ and $f^{\prime}(x) \geq 0$ on $(1, \infty)$. As

$$
f^{\prime}(x)=\frac{p(1-p)\left(x^{1-p}-x^{-p}\right)}{x}-\frac{2 Q(p) \log x}{x},
$$

after substituting $y=x^{p}>0$, it is enough to see that the derivative of

$$
g(y):=x f^{\prime}(x)=p(1-p)\left(y^{\frac{1}{p}-1}-\frac{1}{y}\right)-\frac{2}{p} Q(p) \log y
$$

is non-negative, that is

$$
g^{\prime}(y)=\frac{(1-p)^{2} y^{1 / p}-p\left(\frac{1-p}{p}\right)^{2 p} y+p(1-p)}{y^{2}} \geq 0 \quad \text { for } y>0 .
$$

Hence we need the non-negativity of

$$
h(y):=y^{2} g^{\prime}(y)=(1-p)^{2} y^{1 / p}-p\left(\frac{1-p}{p}\right)^{2 p} y+p(1-p) .
$$

We can obtain the required result from the facts

$$
\begin{gathered}
h^{\prime}(y)=\frac{(1-p)^{2}}{p} y^{\frac{1}{p}-1}-p\left(\frac{1-p}{p}\right)^{2 p}, \quad h^{\prime \prime}(y)=\frac{(1-p)^{3}}{p^{2}} y^{\frac{1}{p}-2}>0, \\
h^{\prime}\left(\left(\frac{p}{1-p}\right)^{2 p}\right)=h\left(\left(\frac{p}{1-p}\right)^{2 p}\right)=0,
\end{gathered}
$$

which altogether mean that $h(y)$ is convex and its minimum is 0 .

We remark that in formula $(2.1), Q(p)=Q(1-p)$ as

$$
Q(1-p)=\frac{(1-p)^{2}}{2}\left(\frac{p}{1-p}\right)^{2-2 p}=\frac{p^{2}}{2}\left(\frac{1-p}{p}\right)^{2 p}=Q(p) .
$$

We can also draw the following consequence of (2.1):

$$
\left(1+R(p)(\log a-\log b)^{2}\right) a^{p} b^{1-p} \leq p a+(1-p) b,
$$

where $R(p)=\min \left\{\frac{p^{2}}{2}, \frac{(1-p)^{2}}{2}\right\}$. This can be easily obtained from the relation $Q(p) \geq R(p)$, which stands, since for $0<p \leq 1 / 2$,

$$
Q(p)=\frac{p^{2}}{2}\left(\frac{1-p}{p}\right)^{2 p} \geq \frac{p^{2}}{2}
$$


and for $1 / 2 \leq p<1$,

$$
Q(p)=Q(1-p) \geq \frac{(1-p)^{2}}{2} .
$$

We finally show that in some cases our inequality (2.1) is better and in other cases is worse than inequality (1.2). For example, for $p=0.4$ and $x=10$,

$$
\begin{aligned}
1.557 \approx & \left(\frac{11^{2}}{40}\right)^{0.4}=K^{0.4}(10)<1+Q(0.4) \log ^{2} 10 \\
& =1+\frac{0.4^{2}}{2}\left(\frac{0.6}{0.4}\right)^{0.8} \log ^{2} 10 \approx 1.586
\end{aligned}
$$

and for $p=0.4$ and $x=30$,

$$
2.298 \approx\left(\frac{31^{2}}{120}\right)^{0.4}=K^{0.4}(30)>1+Q(0.4) \log ^{2} 30 \approx 2.280 .
$$

\section{An application}

Let $A$ and $B$ be two positive invertible operators. Let us define as usual the weighted arithmetic mean as

$$
A \nabla_{p} B:=(1-p) A+p B,
$$

the weighted geometric mean as

$$
A \sharp_{p} B:=A^{1 / 2}\left(A^{-1 / 2} B A^{-1 / 2}\right)^{p} A^{1 / 2}
$$

and the relative operator entropy as

$$
S(A \mid B):=A^{1 / 2} \log \left(A^{-1 / 2} B A^{-1 / 2}\right) A^{1 / 2} .
$$

Then we have an operator inequality version of (2.1):

$$
A \sharp_{p} B+K^{*}\left(A \sharp_{p} B\right) K \leq A \nabla_{p} B,
$$

where $K=\sqrt{Q(p)} A^{-1} S(A \mid B)$ and $Q(p)$ is from (2.1).

Proof of (3.1). From (2.1) we get

$$
\sqrt{a}+Q(p) \log (a) a^{p} \log (a) \leq p a+(1-p),
$$

whence for $X=A^{-1 / 2} B A^{-1 / 2}$,

$$
X^{p}+Q(p) \log (X) X^{p} \log (X) \leq p X+(1-p) I .
$$


Multiplying $A^{1 / 2}$ to the above inequality from the left hand side and the right hand side, we obtain

$$
\begin{aligned}
A \sharp_{p} B+ & Q(p) A^{1 / 2} \log \left(A^{-1 / 2} B A^{-1 / 2}\right) A^{-1 / 2}\left(A \sharp_{p} B\right) A^{-1 / 2} \\
& \times \log \left(A^{-1 / 2} B A^{-1 / 2}\right) A^{1 / 2} \leq p B+(1-p) A,
\end{aligned}
$$

so we have got (3.1).

\section{References}

[1] S. S. Dragomir, On New Refinements and Reverses of Young's Operator Inequality, available online at https://arxiv.org/pdf/1510.01314v1.

[2] S. Furuichi, Refined Young inequalities with Specht's ratio, J. Egyptian Math. Soc., 20 (2012), 46-49.

[3] W. Specht, Zur Theorie der elementaren Mittel, Math. Z., 74 (1960), 91-98.

[4] L. Zou and Y. Jiang, Improved arithmetic-geometric mean inequality and its application, J. Math. Inequal., 9 (2015), 107-111.

[5] G. Zuo, G. Shi and M. Fujii, Refined Young inequality with Kantorovich constant, J. Math. Inequal., 5 (2011), 551-556. 